\section{Good Outcome in a Patient Treated for Cerebral Amyloid Angiopathy Presenting as an Expansive Process with Inflammation and Contrast Enhancement}

Cerebral amyloid angiopathy (CAA) is an important cause of cerebrovascular diseases because it can be treated. CAA may present as a cerebral tumor, but knowledge about the pathophysiology and clinical outcome in such cases is limited. A patient who developed rapid progressive gait disturbances due to CAA with giant cell vasculitis presented with a contrast-enhancing mass lesion on MR imaging. The gait difficulties and the mass lesion normalized after immunosuppressive treatment, while the contrast enhancement persisted.

We illustrate this case of a 57-year-old man who gradually developed weakness in the left leg after a fall on an icy road. After a few weeks, he could not walk without assistance and needed hospitalization at a nursing home. On hospitalization, T1-weighted MR imaging showed an expansive process in the right hemisphere with meningeal contrast enhancement and an old infarction in the left occipital area (Fig 1A). A T2weighted gradient-echo series revealed numerous small punctuate lesions representing microbleeding, indicating amyloid angiopathy, but this was first acknowledged after the diagnosis was proved histologically (Fig 1B). Suspecting a malignant cerebral tumor (glioma) with secondary edema, we treated him with intravenous methylprednisolone with temporary improvement of the weakness in the left leg. Cognitive functions and findings of blood samples and CSF analysis were normal.

An open brain biopsy from the right frontal cortex revealed CAA with giant cell activation around the amyloid deposition (Fig $1 C$ ). The patient was treated with intravenous methylprednisolone, $1 \mathrm{~g}$ daily for 3 days, followed by corticosteroid treatment with a maximum dose of $60 \mathrm{mg}$, tapering off within 3 months. During treatment, he improved, and at the 1-month follow-up consultation, he walked without support and was able to care for himself at home. On examination, he rose from the chair and walked across the room without difficulty. T1-weighted MR imaging showed regression of the mass lesion and almost normalization of the ventricle asymmetry, but meningeal contrast enhancement persisted (Fig $1 D$ ). Four months later, he relapsed with recurrent weakness of the left leg and unsteady gait. Consequently, we administered intravenous cyclophosphamide, $1200 \mathrm{mg}$ at 3-week intervals for 1 year, which resolved the neurologic deficits. During the treatment period and at 15-month follow-up, he remained stable.

Outcome in patients with cerebral mass effect due to CAA-related inflammation is infrequently reported in the literature. ${ }^{1}$ The dramatic regression of the mass lesion demonstrated on MR imaging corresponded well with clinical improvement. Oral prednisolone with or without initial methylprednisolone or cyclophosphamide is the most commonly used immunosuppressive therapy in individual series, and recovery from neurologic deficits has been reported for up to 9 months. ${ }^{2,3}$ In contrast, 1 patient with granulomatous angiitis and a CAA-related mass lesion improved and remained stable for 13 years after surgical excision of the lesion. ${ }^{4}$ After we introduced cyclophosphamide, our patient remained stable for 15 months.

Another case study with review of the literature showed that contrast enhancement was absent in most cases. ${ }^{5}$ As to whether contrast enhancement may reflect inflammatory activity and thereby indicate favorable outcome of immunosuppressant therapy is a matter of speculation, but in a series of patients with related inflammation without expansivity, contrast enhancement was associated with good treatment response. ${ }^{6}$

\section{References}

1. Eng JA, Frosch MP, Kyungchan C, et al. Clinical manifestations of cerebral amyloid angiopathy-related inflammation. Ann Neurol 2004;55:250-56

2. Briceno CE, Resch L, Bernstein M. Cerebral amyloid angiopathy presenting as a mass lesion. Stroke 1987;18:234-39

3. Murphy MN, Sima AA. Cerebral amyloid angiopathy associated with giant cell arteritis: a case report. Stroke 1985;16:514-17

4. Le Coz LP, Mikol J, Ferrand J, et al. Granulomatous angiitis and cerebral amyloid angiopathy presenting as a mass lesion. Neuropathol Appl Neurobiol 1991;17:149-55

5. Safriel Y, Sze G, Westmark K, et al. MR spectroscopy in the diagnosis of cerebral amyloid angiopathy presenting as a brain tumor. AJNR Am J Neuroradio 2004;25:1705-08

6. Salvarani C, Brown RD, Calamia KT, et al. Primary central nervous system vasculitis with prominent leptomeningeal enhancement. Arthritis Rheum 2008;58:595-603

S.I. Bekkelund

C.E. Midtbu

Department of Neurology

L. Arvola

O.P. Eldevik

Department of Radiology

S. Lindal

Department of Pathology

University Hospital of North Norway Institute of Clinical Medicine

University of Tromsø

Tromsø, Norway

DOI 10.3174/ajnr.A2474
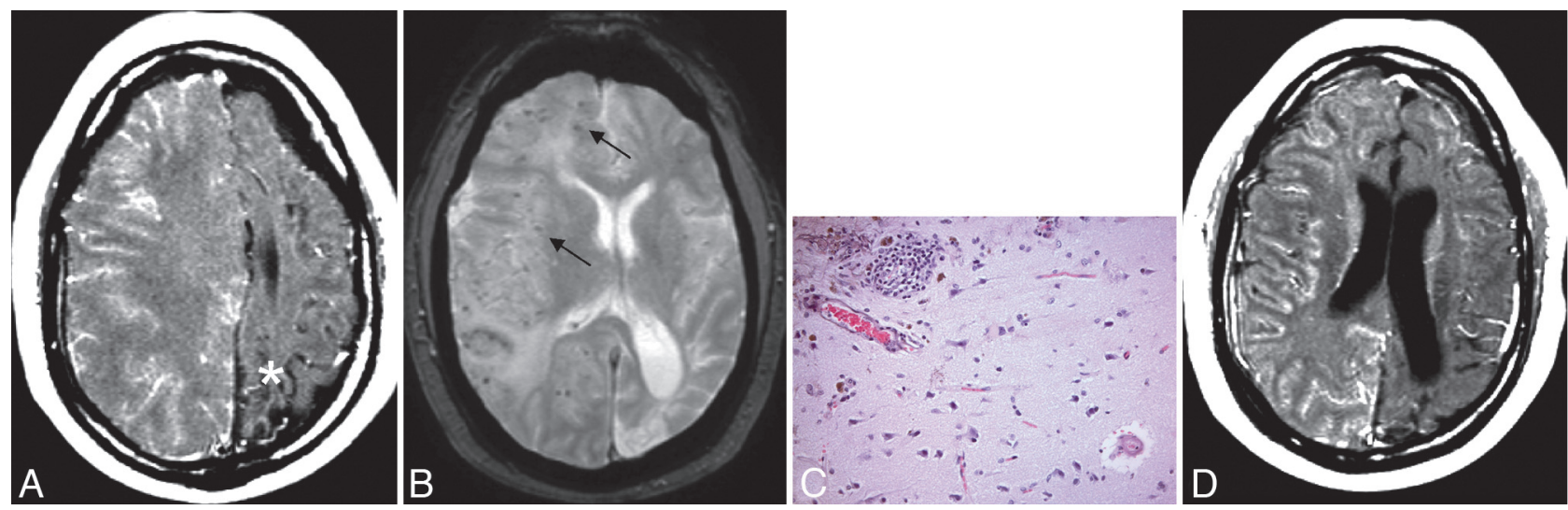

Fig 1. MR imaging in a patient with CAA and vasculitis. $A$, Significant mass effect of the entire right hemisphere and some enhancement of gadolinium of the arachnoid and pia mater on the right side are seen. Note an old infarction in the left occipital lobe (asterisk). B, Punctuate low-signal-intensity lesions (small arrows) in the right hemisphere represent microbleeding, which indicate smyloid angiopathy. C, Amyloid angiopathy with giant cells surrounding the amyloid. A cortical vessel is infiltrated with mononuclear white blood cells and some granulocytes (upper left). Congo red coloring reveals amyloid in 2 of the vessels (upper left and lower right vessel surrounded by edema). Pigmentation reflects hemosiderin deposits from earlier bleeding, while extravasations of red blood cells indicate recent microbleeding. D, MR image after corticosteroid treatment shows normalization of signal-intensity changes in the right frontal lobe and regression of the mass effect. 